\title{
ALGORITHM FOR SOLVING THE INVERSE PROBLEMS OF ECONOMIC ANALYSIS IN THE PRESENCE OF LIMITATIONS
}

\author{
Ekaterina Gribanova \\ Department of Automated Control System \\ Tomsk state university of control systems and radioelectronics \\ 40 Lenina str., Tomsk, Russia, 634050 \\ geb@asu.tusur.ru
}

\begin{abstract}
The solution of inverse problems is considered taking into account the restrictions using inverse calculations. An algorithm is proposed for solving the inverse problem, taking into account restrictions while minimizing the sum of the absolute values of the changes in the arguments. The problem of determining the increments of the function arguments is presented as a linear programming problem. The algorithm includes solving the inverse problem with the help of inverse calculations while minimizing the sum of the absolute changes in the arguments, checking the correspondence of the obtained arguments to the given restrictions, adjusting the value of the argument if it goes beyond the limits of acceptable values, and changing the varied arguments to achieve the given value of the resulting indicator. The solution of two problems with the additive and mixed dependence between the arguments of the function is considered. It is shown that the solutions obtained in this case are consistent with the result of using an iterative procedure based on changing the resulting value to a small value until a given result is achieved, and the results are compared with solving problems using the MathCad mathematical package. The advantage of the algorithm is a smaller number of iterations compared to the known method, as well as the absence of the need to use coefficients of relative importance. The presented results can be used in management decision support systems.
\end{abstract}

Keywords: inverse calculations, inverse problem, linear programming, economic analysis, optimization algorithm.

DOI: $10.21303 / 2461-4262.2020 .001102$

\section{Introduction}

Tasks to be solved in the field of economics can be divided into direct and reverse in the direction of causal relationship. The direct task is establishing an investigation for well-known reasons, i. e., to calculate the result based on the available values of its values and the type of dependence between them. This allows to evaluate the current state of the investigated object, make a forecast for future periods, and study the influence of factors on the output value. An example of solving a direct problem can be the determination of the profit of an enterprise for given values of income and expenses, the determination of revenue for given values of price and quantity.

The inverse problem is to establish the causes leading to the corollary of interest, i. e., such a selection of initial values that would ensure a given value of the result. Such problems are considered incorrect [1] and have become widespread in various fields, including the economy [2-6].

In [7], an inverse computation apparatus is proposed that allows solving an inverse problem using expert information. Solving problems of this kind is relevant, because it allows to answer the question "how to do it so that ...?", determine the control actions to achieve the desired state of the economy, which is an integral function of decision support systems. The inverse computing apparatus has found practical application in various sectors of the economy [8-11].

Solving inverse problems using inverse calculations is to obtain the point values of the growth of the arguments of a function based on its given value and additional information coming from the person who forms the solution. In particular, the coefficients of the relative importance of the argument and the direction of their change (increase or decrease) can be indicated as such information.

The relationship of indicators can be represented in the form of a tree, at the first level of which the resulting indicator is located, at the second - indicators that form it. Fig. 1 shows a graphical illustration of the direct and inverse problems in the case of multiple dependence for the function of two arguments: profitability (r) is equal to the ratio of profit $(p)$ to cost $(c)$ [12]. 
Let's consider the solution of the inverse problem. Initial data: $r=0.2, p=10$ (c.u.), $c=50$ (c.u.). It is necessary to determine the values of profit and costs that will ensure a profitability of 0.3 . Without additional information, this problem can have set of solutions.

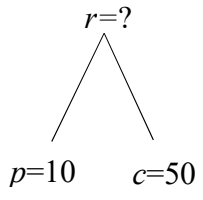

$a$

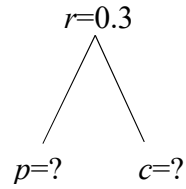

$b$

Fig. 1. Graphical representation of the problem: $a$-direct; $b$-indirect

Let $x_{i}-i$-th argument of the function $f(x)\left(i=1 . . n, n-\right.$ the number of arguments), and $\alpha_{i}-$ the coefficient of relative priority of the $i$-th argument. Then the solution of the problem using inverse calculations can be obtained by solving the system of equations:

$$
\left\{\begin{array}{l}
y \pm \Delta y=f\left(\begin{array}{l}
x_{1} \pm \Delta x_{1}\left(\alpha_{1}\right), x_{2} \pm \\
\pm \Delta x_{2}\left(\alpha_{2}\right), \ldots, x_{n} \pm \Delta x_{n}\left(\alpha_{n}\right)
\end{array}\right) \\
\frac{\Delta x_{1}}{\Delta x_{2}}=\frac{\alpha_{1}}{\alpha_{2}} ; \ldots \frac{\Delta x_{1}}{\Delta x_{n}}=\frac{\alpha_{1}}{\alpha_{n}} ; \\
\sum_{i=1}^{n} \alpha_{i}=1
\end{array}\right.
$$

where $\Delta x_{i}$ - the increment of the i-th argument; $x_{i}$ - the initial value of the i-th argument; $y, \Delta y-$ the initial value and the increment of the resulting function.

So, for problem (1), let's set the values of the coefficients of relative priority of the function arguments: $\alpha_{1}=0.6$ and $\alpha_{2}=0.4$, and an increase in profit and a decrease in costs should occur.

Then the solution to the problem can be obtained as follows:

$$
\begin{gathered}
\left\{\begin{array}{l}
r+\Delta r=\frac{(p+\Delta p)}{(c+\Delta c)} \\
\frac{\Delta p}{\Delta c}=-\frac{\alpha_{1}}{\alpha_{2}}
\end{array}\right. \\
(p-1.5 \Delta c) /(c+\Delta c)=0.3 \\
\Delta c=-2.78 \\
\Delta p=-1.5 \cdot 2.78=4.17
\end{gathered}
$$

The values of profit and costs are equal: $p=10+4.17=14.17, \mathrm{c}=50-2.78=47.22$.

The binding to the expert opinion has its positive aspects: several possible solutions to the problem can be considered, the best change in indicators is indicated from the point of view of available resources, the coefficients can be set taking into account the real possibility of the direction of the change in the arguments and their interdependence. The resulting decision can subsequently be adjusted taking into account additional conditions. However, sometimes it becomes necessary to obtain a result without involving expert information. The decision obtained using the coefficients of relative priority of the arguments is subjective and based on the opinion of a specialist, the determination of which may be difficult. The tasks, the solution of which can be found 
without involving expert information, in particular, include tasks of searching as close as possible to the original solution, i. e., with a minimum change in the arguments. Earlier, two methods for solving such problems were considered.

The first method is considered in [13]; the problem of determining the increments of arguments is represented as the optimization problem of quadratic programming: the sum of the squares of the increments of the arguments acts as the objective function. Using geometric constructions, a method for solving such a problem using a system of equations is determined. However, the scope of this method is limited due to the fact that the dependence function between the arguments can't always be formed. In addition, this method minimizes the sum of the squares of the changes in the arguments, while the absolute values of the arguments can be considered as easier to understand the characteristics of their changes. The sum of the absolute values of the increments of the arguments can be considered as an alternative to the Euclidean distance and suggests a decrease in the influence of large deviations.

The second method is presented in [14] and is based on the concept of a gradient vector, showing the direction of the greatest increase in the function. Therefore, moving in this direction, it is possible to achieve a given value of the function (if necessary, increase it) with a smaller change in the arguments. This method does not require the formation of a function of dependence between arguments, however, it is heuristic and in the problem under study does not guarantee an optimal solution from the point of view of the chosen criterion.

In addition, when solving problems, restrictions on the values of the arguments can be imposed. Restrictions on the values of indicators can be determined by the area of their acceptable values, the features of doing business (for example, the amount of resources used by an enterprise can't be more than the available stock), forecast values of indicators established by an expert, etc.

In [15], an algorithm is proposed for solving the problem in the presence of restrictions, including the gradual change of the resulting indicator to a small step value and the determination of the argument values using inverse calculations using the coefficients of the relative priority of the arguments. If the values of the arguments do not satisfy the constraints, then, if possible, the deficit is filled by other resources. The algorithm ends when the objective function is equal to the specified value or all resources are exhausted.

In [16], stochastic algorithms for solving the inverse problem under constraints were considered. In the first version, the global optimization problem is considered, in which the integral function of the degree of correspondence of the resulting value to the established one and the growth of arguments to the given importance factors acts as the objective function. An algorithm is also proposed based on the sequential change of the resulting indicator by a small amount and the choice of the argument with a probability corresponding to the priority coefficient, due to which a new value of the resulting indicator is achieved. The algorithm stops when it reaches the set value of the function or if all arguments are assigned markers, according to which their further change is impossible.

The disadvantage of the considered work is the need for multiple iterations, therefore, to solve the problem requires a lot of computing resources. In addition, in the case of stochastic algorithms, the resulting solution will be suboptimal.

The presented work is devoted to the development of an algorithm for solving the inverse problem with a minimum total absolute change in the arguments, taking into account the limitations. Unlike existing works, this algorithm will not require multiple solutions to the inverse problem with a sequential approximation of the initial value of the resulting indicator to the given one, the maximum number of iterations will be equal to the number of arguments whose values are constrained.

The aim of research is development of an algorithm for solving inverse problems while minimizing the sum of the absolute values of the arguments, taking into account the limitations that differ from the existing ones, using the approach based on the choice of variable arguments, solving the inverse problem and adjusting the values of the arguments in accordance with the restrictions.

To achieve the aim, the following objectives are set:

- develop a method for solving the inverse problem while minimizing the sum of the absolute values of the arguments; 
- develop an algorithm for solving the inverse problem, taking into account restrictions;

- to compare the solutions obtained as a result of the implementation of the algorithm with the results of using the methods presented in the literature and the mathematical package MathCad.

Thus, the proposed algorithm is based on the use of the inverse computing apparatus proposed in $[7,15]$. It is modified to solve problems without involving expert information (with a minimum amount of absolute values of the arguments), taking into account restrictions on the values of the arguments.

\section{Solution of the problems of economic analysis, taking into account the limitations}

The problem of determining a solution with a minimum sum of the absolute values of the argument changes in the presence of restrictions has the form:

$$
\begin{gathered}
\left|\Delta x_{1}\right|+\left|\Delta x_{2}\right|+\ldots \rightarrow \min , \\
f(x+\Delta x)=y, \\
l_{i} \leq x_{i}+\Delta x_{i} \leq h_{i},
\end{gathered}
$$

where $i$ - the number of the argument, the value of which is constrained (i=1...m, $m$ - the number of arguments the value of which is constrained); $l_{i}$ and $h_{i}-$ the lower and upper bounds of the interval to which the values of the $i$-th argument must belong.

To solve this problem, let's consider an approach that consists in solving the linear programming problem with one constraint $(f(x+\Delta x)=y)$ and then adjusting the solution in accordance with the established boundaries $l_{i}$ and $h_{i}$.

Let's consider a solution to the linear programming problem with one restriction. Depending on the type of model and the increase in the result, the arguments of the objective function (2) will show either a positive or negative sign when the module is opened. So, for example, in the case of the additive model $\left(f(x)=x_{1}+x_{2}\right)$, an increase in the result with a smaller amount of absolute changes in the arguments will be achieved with positive changes in the arguments.

After the module is expanded in the case of a linear constraint $\left(f(x+\Delta x)=b_{1}\left(x_{1}+\Delta x_{1}\right)+\right.$ $\left.+b_{2}\left(x_{2}+\Delta x_{2}\right)+\ldots+b_{n}\left(x_{n}+\Delta x_{n}\right)\right)(b-$ the numerical values for the arguments in the constraint) the problem (2) is a linear programming problem [17]. The classic method for solving it is the simplex method. However, with the only restriction and equality of numerical values for arguments in the objective function to unity, the problem can be solved by a simpler method. Its solution is reduced to finding an element with a large absolute numerical value $b$ with an increase in the argument in the constraint and solving the equation for this argument [18].

If there are several such maximum values, then when solving the problem, either a uniform change of these arguments can be performed to achieve a given value of the result, or their change in accordance with the coefficients of relative importance specified by the expert, or a change in one of the arguments chosen randomly. When implementing software systems, the equation is solved using classical methods for finding roots (for example, by the Newton method), the article discusses the analytical solutions to such problems.

If the restriction has a nonlinear form, the considered problem relates to nonlinear programming problems [19]. To convert the constraint into a linear one, Taylor series expansion can be used (the initial values of the increments are zero):

$$
f\left(\Delta x_{1}, \Delta x_{2}, \ldots, \Delta x_{n}\right)=\sum_{i=1}^{n} \frac{\partial f}{\partial \Delta x_{i}} \Delta x_{i} .
$$

Consequently, the value of the partial derivatives $\left(b_{i}=\frac{\mathrm{d} f}{\mathrm{~d} \Delta x_{i}}\right)$ at the zero point is considered as numerical values in increments of the arguments. 
Thus, the algorithm for determining the solution of the inverse problem using inverse computation, if there are restrictions on the values of the arguments, includes the following steps.

Step 1. Solving the problem of determining the increments $\Delta x_{i}$ of variable arguments in such a way that the sum of their absolute values is minimal:

$$
\begin{gathered}
\left|\Delta x_{1}\right|+\left|\Delta x_{2}\right|+\ldots \rightarrow \min \\
f(x+\Delta x)=y
\end{gathered}
$$

Change the argument values by the obtained increment value: $x_{i}=x_{i}+\Delta x_{i}$.

Step 2. Verify compliance of mutable arguments with given constraints. If all values satisfy the constraints, then the algorithm stops. If there is at least one argument that does not satisfy the restrictions, then the value of the nearest boundary is assigned to it, and such an argument is excluded from the list of mutable arguments. If the list of mutable arguments is empty, then the algorithm ends.

In the presented algorithm, in step 1, the solution of problem (4) occurs, however, minimization of the sum of the squares of the increments and the change in the increments of the arguments in accordance with the elements of the gradient vector can be considered. This algorithm will allow to find a solution to such problems in the presence of restrictions.

\section{The results of solving inverse problems with restrictions}

Let's consider the application of the described algorithm for the additive and mixed dependence between the arguments of a function. Relative priority coefficients are excluded from the tasks.

The volume of delivery $Q$ of products to the outlet consists of the delivery volume of the first, second and third type of product:

$$
Q=Q_{1}+Q_{2}+Q_{3} \text {, }
$$

where $Q_{1}, Q_{2}, Q_{3}$ - the volume of delivery of products of the first, second and third kind, respectively.

The initial values of the arguments are: $Q_{1}=15 \mathrm{~kg}, Q_{2}=17 \mathrm{~kg}, Q_{3}=20 \mathrm{~kg}$. The initial value of the resulting indicator $Q$ is $52 \mathrm{~kg}$. It is necessary to determine the volume of delivery of products of the first, second and third type so that the total volume is $60 \mathrm{~kg}$ of products.

At the same time, the following restrictions are set for argument values:

$$
\begin{aligned}
& 0 \leq Q_{1} \leq 25 ; \\
& 0 \leq Q_{2} \leq 17.5 ; \\
& 0 \leq Q_{3} \leq 25 .
\end{aligned}
$$

Let's carry out the solution of the inverse problem with the help of inverse calculations under given constraints. Since the value of the resulting function needs to be increased, this can be achieved with positive gains in these arguments. The task has the form:

$$
\begin{gathered}
\Delta Q_{1}+\Delta Q_{2}+\Delta Q_{3} \rightarrow \min \\
\left(Q_{1}+\Delta Q_{1}\right)+\left(Q_{2}+\Delta Q_{2}\right)+\left(Q_{3}+\Delta Q_{3}\right)=60 \\
\Delta Q_{1}, \Delta Q_{2}, \Delta Q_{3} \geq 0 .
\end{gathered}
$$

The numerical values for $\Delta Q_{1}, \Delta Q_{2}$ and $\Delta Q_{3}$ are equal. Let's consider the case when the change in the growth of arguments occurs evenly. 
The system of equations has the form:

$$
\left\{\begin{array}{l}
\frac{\Delta Q_{1}}{\Delta Q_{2}}=1, \\
\frac{\Delta Q_{1}}{\Delta Q_{3}}=1, \\
\left(15+\Delta Q_{1}\right)+\left(17+\Delta Q_{2}\right)+\left(20+\Delta Q_{3}\right)=60 .
\end{array}\right.
$$

System solution:

$$
\Delta Q_{1}=\Delta Q_{2}=\Delta Q_{3}=2.667
$$

Therefore,

$$
\begin{gathered}
Q_{1}=15+2.667=17.667, \\
Q_{2}=19.667, Q_{3}=22.667 .
\end{gathered}
$$

The argument $Q_{2}$ does not satisfy the restrictions; let's assign this argument the value of the boundary $17.5\left(Q_{2}=17.5\right)$. The list of mutable arguments left $Q_{1}$ and $Q_{3}$.

The system of equations has the form:

$$
\left\{\begin{array}{l}
\frac{\Delta Q_{1}}{\Delta Q_{3}}=1, \\
\left(17.667+\Delta Q_{1}\right)+17.5+\left(22.667+\Delta Q_{3}\right)=60 .
\end{array}\right.
$$

System solution:

$$
\Delta Q_{1}=\Delta Q_{3}=1.083
$$

Therefore,

$$
\begin{gathered}
Q_{1}=17.667+1.083=18.75, Q_{2}=17.5, \\
Q_{3}=22.667+1.083=23.75 .
\end{gathered}
$$

The solution corresponds to the obtained solution using the MathCad program (Fig. 2).

$$
\begin{aligned}
& \Delta \mathrm{Q} 1:=0 \quad \Delta \mathrm{Q} 2:=0 \quad \Delta \mathrm{Q} 3:=0 \quad \mathrm{Q} 1:=15 \quad \mathrm{Q} 2:=17 \quad \mathrm{Q} 3:=20 \\
& \mathrm{f}(\Delta \mathrm{Q} 1, \Delta \mathrm{Q} 2, \Delta \mathrm{Q} 3):=|\Delta \mathrm{Q} 1|+|\Delta \mathrm{Q} 2|+|\Delta \mathrm{Q} 3| \\
& \text { Given } \\
& 14 \leq \mathrm{Q} 1+\Delta \mathrm{Q} 1 \leq 2516 \leq \mathrm{Q} 2+\Delta \mathrm{Q} 2 \leq 17.518 \leq \mathrm{Q} 3+\Delta \mathrm{Q} 3 \leq 25 \\
& (\mathrm{Q} 1+\Delta \mathrm{Q} 1)+(\mathrm{Q} 2+\Delta \mathrm{Q} 2)+(\mathrm{Q} 3+\Delta \mathrm{Q} 3)=60 \\
& \Delta \mathrm{Q}:=\operatorname{Minimize}(\mathrm{f}, \Delta \mathrm{Q} 1, \Delta \mathrm{Q} 2, \Delta \mathrm{Q} 3) \\
& \mathrm{Q} 1:=\mathrm{Q} 1+\Delta \mathrm{Q}_{0}=18.75 \\
& \mathrm{Q} 2:=\mathrm{Q} 2+\Delta \mathrm{Q}_{1}=17.5 \\
& \mathrm{Q} 3:=\mathrm{Q} 3+\Delta \mathrm{Q}_{2}=23.75
\end{aligned}
$$

Fig. 2. Problem solution in MathCad 
Fig. 3 shows the change in the values of the arguments during ten iterations when solving the problem using the iterative procedure presented in [15]. The result obtained corresponds to the solution in Fig. 2.

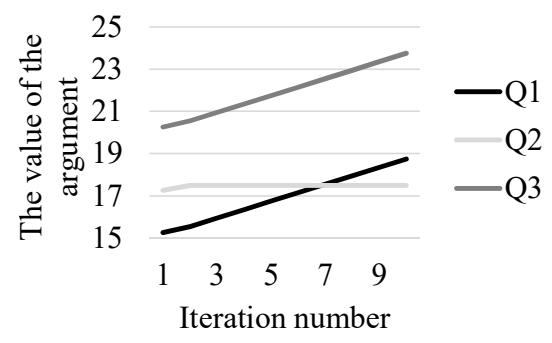

Fig. 3. Changing argument values over ten iterations

Let's consider the mixed dependency between function arguments. Profit $(M)$ of an enterprise is defined as the difference between income and costs [20, 21]:

$$
M=N u m(\text { Price }-C u) \text {, }
$$

where Price - the unit price of a product; Num - amount of product sold; $\mathrm{Cu}$ - unit cost of a product.

The initial value of the profit from the sale of products of the type in question is 4,018 c.u., the unit price is 60 c.u., the quantity is 98 units, the unit cost of production is 19 c.u. It is necessary to determine the value of the price, cost and quantity in this way so that the profit is equal to $4500 \mathrm{c} . \mathrm{u}$.

Constraints for variables: $80 \leq N u m \leq 130,50 \leq$ Price $\leq 62.5,18.5 \leq C u \leq 25$.

Let's calculate the partial derivatives of the constraint function $f(\Delta N u m, \Delta$ Price, $\Delta C u)=$ $=(98+\Delta N u m) \cdot(60+\Delta$ Price $-19-\Delta C u)-4500$ :

$$
\begin{gathered}
\frac{\partial f}{\partial \Delta N u m}=41, \\
\frac{\partial f}{\partial \Delta \text { Price }}=98, \\
\frac{\partial f}{\partial \Delta C u}=-98 .
\end{gathered}
$$

The absolute value of the partial derivative is greater for price and cost increment. Let's consider the uniform change in these indicators:

$$
\left\{\begin{array}{l}
\frac{\Delta \text { Price }}{\Delta C u}=-1 ; \\
98 \cdot(60+\Delta \text { Price }-19-\Delta C u)=4500
\end{array}\right.
$$

System solution: $\Delta$ Price $=2.459, \Delta C u=-2.459$, Price $=62.459, C u=16.54$. The cost value does not satisfy the limit. Consequently, $C u=18.5$, $\Delta$ Price $=4500 / 98-62.459+18.5=1.959$.

The value of the price does not satisfy the restriction, therefore,

$$
\text { Price }=62.5
$$

$N u m=4500 /(62.5-18.5)=102.273$.

The resulting value satisfies the constraint, the calculations are completed. 
The solution also coincided with the solution of problem (1) in the MathCad program.

Table 1 presents the results obtained using the considered algorithm in the case of minimizing the sum of the squares of the changes in the arguments.

Table 1

Argument values while minimizing the squared argument changes

\begin{tabular}{cccc}
\hline Iteration number & Num & Price & Cu \\
\hline & 98 & 60 & 19 \\
2 & 99.02 & 62.223 & 16.777 \\
3 & 99.667 & 63.65 & 18.5 \\
& 102.273 & 62.5 & 18.5
\end{tabular}

4. Discussion of the results of the development of an algorithm for solving inverse problems with constraints

An algorithm is proposed for solving the inverse problem using inverse calculations while minimizing the sum of the absolute changes in the arguments, taking into account restrictions on the values of the arguments. The results of applying the algorithm are consistent with the solutions obtained using the mathematical package and applying the iterative procedure presented in [15].

The advantage of the proposed algorithm is that, unlike the approaches considered in $[15,16]$, it does not require multiple iterations, during which the resulting indicator is successively changed until a given value is reached. In the proposed algorithm, the number of iterations is determined by the number of arguments that do not satisfy the constraints. The algorithm, in contrast to the method proposed in [13], which considers minimizing the sum of the squares of the arguments, does not require the formation of a function of dependence between the arguments and suggests finding a solution while minimizing the absolute deviations of the arguments (a task in such a formulation may be more understandable for a specialist).

The disadvantages of the proposed method include the need to calculate partial derivatives of the function in the case of a nonlinear dependence between the arguments of the function.

The presented algorithm does not require the use of the simplex method and can be used to solve linear programming problems of the considered type in other areas of research. The disadvantage of the proposed algorithm is the requirement that the number of constraints that include different variables should be equal to unity, which narrows the range of tasks.

The directions of further research are related to the modification of this algorithm for the case of discrete data, i. e., if there is a requirement that the arguments of the function are integer.

\section{Conclusions}

1. An algorithm is proposed for solving the inverse problem while minimizing the sum of the absolute increments of the arguments, taking into account restrictions on the values of the arguments. The application of the algorithm allows one to obtain results that are consistent with the results of using mathematical packages and an iterative method, which involves changing the resulting indicator by a small amount until the specified value is reached. Confirmation of this is given in the results of the numerical solution of two inverse problems in which the additive and mixed dependence between the arguments is considered. A feature of the algorithm is the absence of the need for multiple iterations, as well as the ability to determine a solution with a minimum total absolute change in the arguments. This is achieved by presenting the problem as a linear programming problem with one restriction. Its solution reduces to determining the maximum absolute numerical values for the arguments in the constraint and solving the inverse problem using inverse calculations.

2. The presented algorithm can be implemented in decision support systems, providing organization specialists with the opportunity to solve inverse problems using inverse calculations if there are restrictions without using expert information. The approach will allow to determine the solution to problems with a minimum total absolute change in the arguments, i. e., the one closest to the current one, and ensure the speed of such software systems. 


\section{References}

[1] Colton, D., Engl, H. W., Louis, A. K., McLaughlin, J. R., Rundell, W. (Eds.) (2000). Surveys on Solution Methods for Inverse Problems. Springer. doi: https://doi.org/10.1007/978-3-7091-6296-5

[2] Ekeland, I., Djitté, N. (2006). An inverse problem in the economic theory of demand. Annales de l'Institut Henri Poincare (C) Non Linear Analysis, 23 (2), 269-281. doi: https://doi.org/10.1016/j.anihpc.2005.10.001

[3] Barbagallo, A., Mauro, P. (2014). An Inverse Problem for the Dynamic Oligopolistic Market Equilibrium Problem in Presence of Excesses. Procedia - Social and Behavioral Sciences, 108, 270-284. doi: https://doi.org/10.1016/j.sbspro.2013.12.837

[4] Shananin, A. A. (2018). Inverse Problems in Economic Measurements. Computational Mathematics and Mathematical Physics, 58 (2), 170-179. doi: https://doi.org/10.1134/s0965542518020161

[5] Klemashev, N. I., Shananin, A. A. (2016). Inverse problems of demand analysis and their applications to computation of positively-homogeneous Konüs-Divisia indices and forecasting. Journal of Inverse and Ill-Posed Problems, 24 (4). doi: https:/doi.org/ 10.1515/jiip-2015-0015

[6] Beck, A., Teboulle, M. (2009). A Fast Iterative Shrinkage-Thresholding Algorithm for Linear Inverse Problems. SIAM Journal on Imaging Sciences, 2 (1), 183-202. doi: https://doi.org/10.1137/080716542

[7] Odintsov, B. E., Romanov, A. N. (2014). How to create business performance management (BPM) system. Bulletin of the University of Finance, 6, 22-36.

[8] Barmina, E. A., Kvjatkovskaja, I. Yu. (2010). Monitoring the quality of a commercial organization. Structuring indicators. The use of cognitive maps. Bulletin of the Astrakhan State Technical University, 2, 15-20.

[9] Blumin, S. L., Borovkova, G. S. (2018). Application of analysis of finite fluctuations and reverse computations in control systems and decision support systems. Control Sciences, 6, 29-34. doi: https://doi.org/10.25728/pu.2018.6.4

[10] Borshchuk, I. V., Odintsov, B. E., Shkvir, V. D. (2013). Inverse calculations in prevent crisis phenomenon of socio-economic systems. International conference on application of information and communication technology and statistics in economy and education. Bulgaria, 140-143.

[11] Dik, V. V., Urintsov, A. I., Odintsov, B. Ye., Churikanova, O. Yu. (2014). Decision support methods in balanced ScoreCard. Naukovyi visnyk Natsionalnoho hirnychoho universytetu, 4, 120-126.

[12] Ali, H. A. E. M., Al-Sulaihi, I. A., Al-Gahtani, K. S. (2013). Indicators for measuring performance of building construction companies in Kingdom of Saudi Arabia. Journal of King Saud University - Engineering Sciences, 25 (2), 125-134. doi: https:// doi.org/10.1016/j.jksues.2012.03.002

[13] Gribanova, E. B. (2018). Methods for solving inverse problems of economic analysis by minimizing argument increments. Proceedings of Tomsk State University of Control Systems and Radioelectronics, 21 (2), 95-99. doi: https://doi.org/10.21293/18180442-2018-21-2-95-99

[14] Gribanova, E. (2019). Development of a price optimization algorithm using inverse calculations. Eastern-European Journal of Enterprise Technologies, 5 (4 (101)), 18-25. doi: https://doi.org/10.15587/1729-4061.2019.180993

[15] Odincov, B. E., Romanov, A. N. (2014). Iterative method of optimization of enterprise management by means of inverse calculations. Bulletin of the Financial University, 2, 60-73.

[16] Gribanova, E. B. (2016). Stochastic algorithms to solve the economic analysis inverse problems with constraints. Proceedings of Tomsk State University of Control Systems and Radioelectronics, 19 (4), 112-116. doi: https://doi.org/10.21293/1818-04422016-19-4-112-116

[17] Vanderbei, R. J. (2014). Linear programming. Foundations and Extensions. Springer. doi: https://doi.org/10.1007/978-1-46147630-6

[18] Ganicheva, A. V. (2019). Method of the solution of some classes optimising tasks. Modeling, optimization and information technology, 7 (2), 43-54. doi: https://doi.org/10.26102/2310-6018/2019.25.2.002

[19] Trunov, A. (2015). Modernization of means for analyses and solution of nonlinear programming problems. Quantitative Methods in Economics, XVI (2), 133-141.

[20] Shen, B., Shen, Y., Ji, W. (2019). Profit optimization in service-oriented data market: A Stackelberg game approach. Future Generation Computer Systems, 95, 17-25. doi: https://doi.org/10.1016/j.future.2018.12.072

[21] O’Neill, B., Sanni, S. (2018). Profit optimisation for deterministic inventory systems with linear cost. Computers \& Industrial Engineering, 122, 303-317. doi: https://doi.org/10.1016/j.cie.2018.05.032

Received date 12.11.2019

Accepted date 16.12.2019

Published date 20.12.2019
(C) The Author(s) 2020

This is an open access article under the CC BY license (http://creativecommons.org/licenses/by/4.0). 